\title{
Current progress of mitochondrial transplantation that promotes neuronal regeneration
}

\author{
Chu-Yuan Chang ${ }^{1}$, Min-Zong Liang ${ }^{1}$ and Linyi Chen ${ }^{1,2^{*}}$ (D)
}

\begin{abstract}
Background: Mitochondria are the major source of intracellular adenosine triphosphate (ATP) and play an essential role in a plethora of physiological functions, including the regulation of metabolism and the maintenance of cellular homeostasis. Mutations of mitochondrial DNA, proteins and impaired mitochondrial function have been implicated in the neurodegenerative diseases, stroke and injury of the central nervous system (CNS). The dynamic feature of mitochondrial fusion, fission, trafficking and turnover have also been documented in these diseases.

Perspectives: A major bottleneck of traditional approach to correct mitochondria-related disorders is the difficulty of drugs or gene targeting agents to arrive at specific sub-compartments of mitochondria. Moreover, the diverse nature of mitochondrial mutations among patients makes it impossible to develop one drug for one disease. To this end, mitochondrial transplantation presents a new paradigm of therapeutic intervention that benefits neuronal survival and regeneration for neurodegenerative diseases, stroke, and CNS injury. Supplement of healthy mitochondria to damaged neurons has been reported to promote neuronal viability, activity and neurite re-growth. In this review, we provide an overview of the recent advance and development on mitochondrial therapy.

Conclusion: Key parameters for the success of mitochondrial transplantation depend on the source and quality of isolated mitochondria, delivery protocol, and cellular uptake of supplemented mitochondria. To expedite clinical application of the mitochondrial transplantation, current isolation protocol needs optimization to obtain high percentage of functional mitochondria, isolated mitochondria may be packaged by biomaterials for successful delivery to brain allowing for efficient neuronal uptake.
\end{abstract}

Keywords: Mitochondrial dynamics, Mitochondrial therapy, Neurodegenerative diseases, Stroke, Neuronal regeneration

\section{Background}

Mitochondria are double-membraned cytoplasmic organelles that generate the majority of adenosine triphosphate (ATP) via oxidative phosphorylation. In addition to energy production, mitochondria also function in the biosynthesis of fatty acids, cellular calcium buffering, and act as a platform to integrate cell signalling circuitry that modulates cell survival, immune response, and autophagy $[1,2]$. It has been hypothesized that mitochondria evolved from engulfed prokaryotic bacteria so that

\footnotetext{
* Correspondence: Ichen@life.nthu.edu.tw

${ }^{1}$ Institute of Molecular Medicine, National Tsing Hua University, 101, Section 2, Kuang-Fu Road, Hsinchu 30013, Taiwan

${ }^{2}$ Department of Medical Science, National Tsing Hua University, Hsinchu 30013, Taiwan
}

they possess their own circular DNA (mitochondrial DNA, mtDNA) encoding 37 genes and 13 mitochondrial proteins. Together with nuclear encoded mitochondrial proteins, they maintain mitochondrial integrity [2-4]. Research in the past decade has unveiled that mitochondria are dynamic bioenergetic organelles undergoing controlled fusion, fission, transport, and targeted turnover. Mitochondrial population and quality are controlled in part by dynamic morphogenesis. Initiation of mitochondrial fission starts with recruiting cytosolic dynamin-related protein 1 (Drp1) to mitochondrial outer membrane and forming Drp1 oligomers at candidate fission site, which is marked by ER-mitochondria contact region. Drp1 oligomers then constrict mitochondrial membrane upon GTP hydrolysis to divide mitochondria 
[5-7]. Fusion, on the other hand, is initiated by mitofusin-1 and -2 (Mfn1 and Mfn2), which are anchored to the outer mitochondrial membrane (OMM) and mediate fusion of OMM. Fusion of inner membrane (IMM) depends on inner membrane GTPase optic atrophy protein 1 (OPA1), which is spliced into long isoform, L-OPA1, and short isoform, S-OPA1. L-OPA1 is required for IMM fusion while S-OPA1 is associated with mitochondrial fission $[5,6]$.

The dynamic feature of mitochondria serves to adjust cellular metabolism according to physiological states $[8$, 9]. During early development, stochastic mitochondrial segregation leads to genetic drift effect, raising the risk of pathogenic homoplasmy and the subsequent mitochondrial dysfunction. Given the maternal inheritance of mtDNA, accumulated mtDNA mutations are very likely to be transmitted to the offspring during fertilization whilst paternal mtDNA is targeted to be destroyed. Consequently, the highly dynamic nature of mitochondria evolves as a compensation to retain mitochondrial heteroplasmy in cells [10]. Mitochondrial fusion requires the fusion of outer and inner mitochondrial membranes to form tubular or elongated interconnecting mitochondrial networks within cells and allows the communication of mitochondrial materials between organelles. As mutated mtDNA accumulates, mitochondrial fusion buffers defective mtDNA by mixing wild-type and mutant mtDNA to compensate mitochondrial function or undergoing mtDNA recombination to prevent homoplasmic inheritance of mutated mtDNA into daughter cells [10]. Mitochondrial fission, in contrast, has mainly been implicated in mitochondrial replication, transport, turnover, and cell survival. During cell division, mitochondria are replicated and split into daughter cells. As part of mitochondrial quality control machinery, mitochondrial fission antagonizes fusion events and prompts segregation of damaged mitochondria for further destruction via mitophagy. Divided smaller mitochondria facilitate mitochondrial transport through interaction with motor proteins along cytoskeletal networks to meet energy demand at distal region. For example, mitochondrial fission and recruitment are prominent in primary cortical neurons during development and in vicinity of dendritic protrusions of hippocampal neurons to benefit the plasticity of spines and synapses [11, 12]. Drp1dependent mitochondrial fission has been reported to modulate programmed cell death following the recruitment of pro-apoptotic proteins, such as $\mathrm{Bcl}$-2-associated $\mathrm{X}$ protein (Bax) and $\mathrm{Bcl}-2$ antagonist. Findings from our laboratory also reveal enhanced mitochondrial fission in response to injury and during regeneration of hippocampal neurons [13].

Brain is highly energy-demanding, consuming about $20 \%$ of body's energy. Thus, mitochondrial localization within dendrites and axons supply energy as well as to maintain calcium homeostasis [14]. It is thus not surprising to find that mitochondrial distribution and transport are essential for synaptogenesis and dendritic spine formation during development as well as for regulating neuronal activity and behaviour [11, 14]. The dependency of neuronal function and structure on mitochondrial integrity and dynamics is echoed by increasing studies that demonstrate mitochondrial dynamic abnormalities in the well documented neurodegenerative diseases, such as Alzheimer's disease (AD), Parkinson's disease (PD), Huntington's disease (HD), ischemic stroke and traumatic brain injury (TBI) [15-17] . To this end, better understanding the mechanism underlying defective mitochondrial dynamics and function in these diseases would provide insights into the improvement of clinical treatment. In this review, we summarize and discuss recent reports that lead to the emerging mitochondrial therapy.

\section{Mitochondrial dynamics and diseases Neurodegenerative diseases}

Due to the complexity and therapeutic setbacks of current treatment for neurodegenerative diseases, increasing attention points to the mitochondria-related pathogenesis $[15,18]$. Reduced utilization of glucose in the brain measured by flurodeoxyglucose positron emission tomography (FDG PET) suggests metabolic defect in AD brain and prompts the exploration of the role of mitochondria in $\mathrm{AD}$ pathogenesis [19]. In AD, increased S-nitrosylation at Cys644 and phosphorylation at Ser616 of Drp1 protein enhance the GTPase activity and lead to mitochondrial fragmentation [20, 21]. Inhibition of Drp1 in AD models restores amyloid beta $(A \beta)$-mediated mitochondrial dysfunction, synapse damage, and cognitive impairment. Increase of mitochondrial fragmentation in $\mathrm{AD}$ subjects could also result from up-regulated fission proteins (Drp1, Fis1) and down-regulated fusion proteins (Mfn1, Mfn2, OPA1) that partially contribute to gradual neuronal loss and synapse impairment [22-26]. In addition, the absence of an autophagy/mitophagy regulator PTEN-induced putative kinase protein 1 (PINK1) on OMM within neurofibrillary tangles of $\mathrm{AD}$ brain fails to recruit Parkin protein upon membrane depolarization and thus underlies the accumulation of damaged mitochondria in AD patients [27]. Intra-hippocampal injection of PINK1-expressing construct to transgenic mice that overexpress human form of mutant amyloid precursor protein effectively alleviates $\mathrm{A} \beta$-mediated mitochondrial dysfunction and rescues the mitophagy defect via recruiting autophagy receptors (nuclear dot protein $52 \mathrm{kDa}$, optineurin) to damaged mitochondria to activate mitophagy signalling [26, 28]. PDassociated leucine-rich repeat kinase 2 (LRRK2) mutant and HD-associated mutant huntingtin protein $(\mathrm{mHtt})$ were found interacting with Drp1 to enhance mitochondrial 
fission, accompanied by defective anterograde mitochondrial transport and synapse degeneration $[29,30]$. The toxicity of mutant PD-associated proteins, including PINK1, Parkin, LRRK2, protein deglycase DJ-1,vacuolar protein sorting-associated protein 35 , and $\alpha$-synuclein, accounts for mitochondrial fission, impaired mitophagy, and neuronal death in the PD genetic models $[15,31]$. Loss of synapses concurred with deficiency of mitochondrial complexes I and IV in PD neurons within substantia nigra (SN) were also observed [32]. For HD patients, $\mathrm{mHtt}$ protein directly or indirectly alters mitochondrial morphology, functions, bioenergetics status, and dynamics, mainly in the striatum and cortical cerebrum [33, 34]. In addition to mHtt-Drp1interaction-mediated mitochondrial fission [35], $\mathrm{mHtt}$ interacts with OMM and leads to defect of calcium homeostasis. High sensitivity of mitochondria to calcium-induced permeability transition pore in $\mathrm{mHtt}$-expressing clonal striatal cells (conditionally immortalized cells of striatal origin) and striatal neurons results in increased calcium release in the presence of ROS stress [36-38]. However, the clearance of defective mitochondria via mitophagy is inhibited due to the binding of $\mathrm{mHtt}$ aggregates to the adaptor proteins, such as p62 and huntingtin-associated protein-1, during formation and transport of autophagosomes [39-41]. Notably, it was demonstrated in HD mice that decreased activity of mitochondrial complex IV and reduced ATP production in striatal cells precede neuronal death [42]. Inhibiting mitochondrial citric acid cycle by administrating 3 -nitropropionic acid in the animal models resembles the pathology and symptomatology in HD [43, 44].

\section{Stroke}

Mitochondrial fission was regarded as an early pathological event in ischemic stroke mice and accompanied by morphological change of mitochondria, high level of free radicals, and ATP depletion [45]. In the middle cerebral artery occlusion (MCAO) mice model, mitochondrial fission occurred in penumbra region $3 \mathrm{~h}$ after reperfusion [45]. Another study showed that oxygenglucose deprivation (OGD)-induced mitochondrial fission resulted in neuronal cell death and inhibition of Drp1 by siRNA or pharmacological inhibitors prevented mitochondrial fission, reduced death of cortical neurons and reduced the infarct volume in ischemic stroke mice [46]. PINK1 was reported to prevent subcellular translocation of Drp1 and reversed mitochondrial fission induced by OGD. Knockdown of PINK1 caused an increase in fragmented mitochondria and worsened the collapse of mitochondrial membrane potential [47]. The MCAO mice and hypoxic/ischemic condition in hippocampal neurons suppressed the expression of Mfn2. Overexpression of Mfn2 increased the ratio of $\mathrm{Bcl}-2 / \mathrm{Bax}$ and reduced the cleaved caspase 3 and cytochrome c release after hypoxia [48]. These studies indicate that the excess of mitochondrial fission induced by stroke leads to mitochondrial damage and cell death. Thus, restoration of the imbalanced mitochondrial dynamics may potentially be a way to attenuate stroke-induced neuronal death.

\section{Traumatic brain injury}

Studies dated back in 1960s revealed increased number of mitochondria following neuro-axotomy of motor neurons $[49,50]$. Mitochondrial swelling were observed in isolated sensory ganglions from limb-amputated newt [51] and in dorsal root ganglions after sciatic nerve crush in rat [52]. Dimova et al. performed axonal section on rat hypoglossal neurons and noted the increased clustering of hypertrophic mitochondria around axon hillock along with strong respiration activity (Fig. 1a and b) [53]. Our previous study reported that fragmented mitochondria were increased $24-48 \mathrm{~h}$ after injury in primary hippocampal neurons [13]. Another study showed reduced length of mitochondria in hippocampal neurons after TBI in a controlled cortical impact (CCI) mouse model. The aberrant mitochondrial fission was caused by the increase in Drp1 translocation but not total Drp1 level. Excessive Drp1-mediated mitochondrial fission in TBI animals impairs mitochondrial respiration, leads to reactive oxygen species (ROS) overproduction, and neuronal loss [16]. Mitochondrial division inhibitor 1 (Mdivi1) treatment attenuated the reduction of mitochondrial length and protected new-born neurons in the hippocampus post injury [16]. A recent study reported that Mdivi-1 blocked the induction of mitochondrial fission and mitophagy in a CCI model of moderate TBI [54]. It appears that TBI induces mitochondrial fission and inhibiting fission can reduce the damage caused by TBI. However, another study on TBI model of rats suggests that the change of mitochondrial fission/fusion dynamics depends on injury severity. The expression level of the genes involved in fission and fusion were downregulated and up-regulated, respectively, following a mild TBI. In contrast, mitochondrial fission was increased following a severe TBI [55]. Due to the complexity of TBI, it remains debatable whether mitochondrial fission enables higher mobility of mitochondria to the injury site for regeneration or is a result of tissue damage. Nevertheless, these two conclusions do not necessarily conflict with each other.

\section{A new paradigm of therapeutic strategy: mitochondrial therapy Mitochondrial dynamics and neuronal regeneration}

As accumulating data demonstrate the interplay between defective mitochondrial biogenesis and diseases, several lines of evidence reveal dynamic morphogenesis during neuronal regeneration. Our laboratory previously reported 


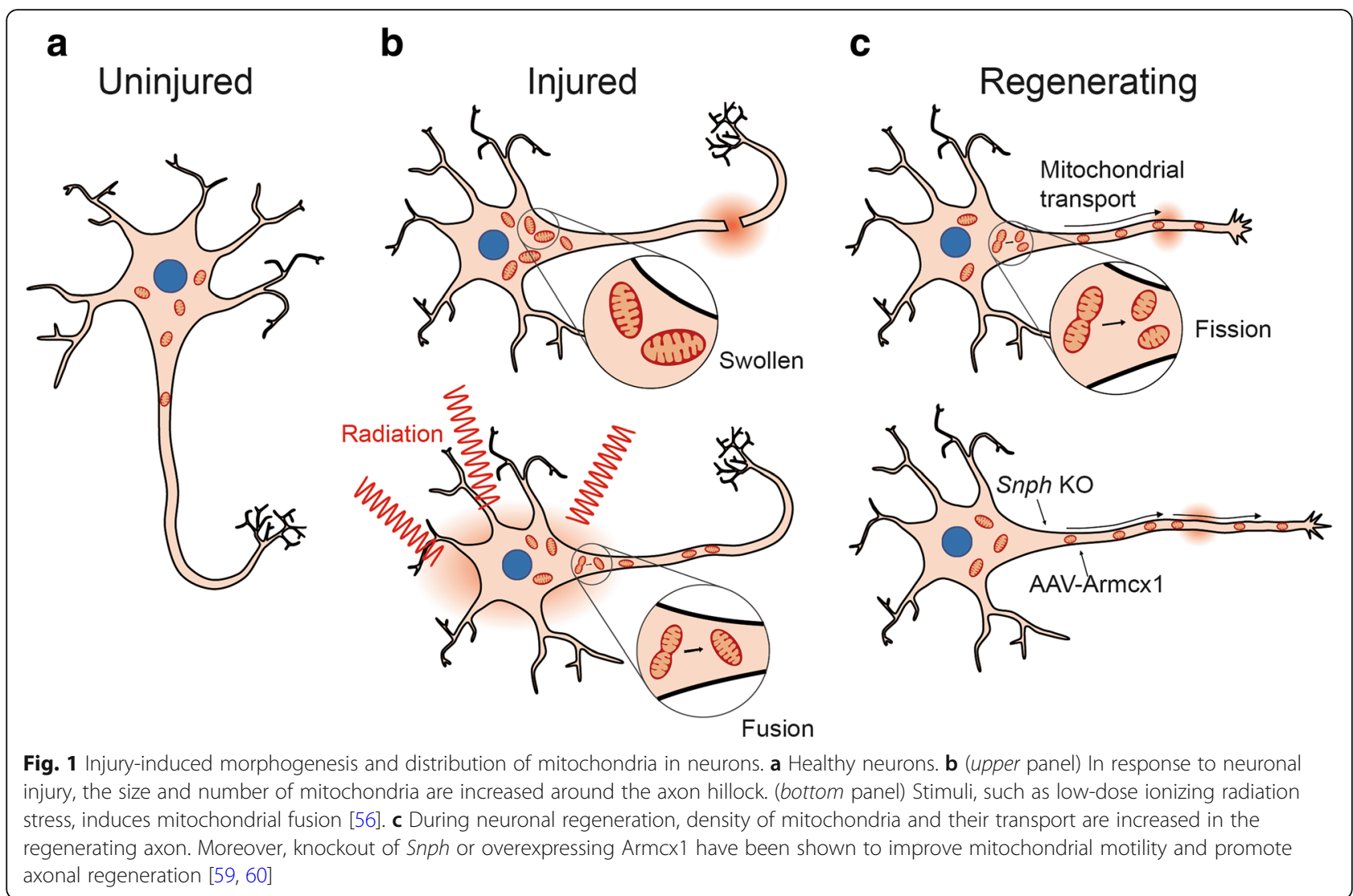

that increased mitochondrial fusion promoted survival of hippocampal neurons in response to low-dose ionizing radiation (Fig. 1b) [56]. Interestingly, in response to TBI, mitochondrial fission was increased in hippocampal neurons allowing faster mobilization of smaller/fragmented mitochondria to the injury site, likely to facilitate regeneration process [13]. Along this line, live cell imaging of regenerating neurons after laser axotomy of $\gamma$-aminobutyric acid motor neurons of C. elegans and Mauthner axons of zebra fish suggests that increased number of mitochondria translocated in injured axons and that mitochondrial mobility is positively correlated with axonal regeneration [57, 58]. Furthermore, genetic knockout of Snph, a gene encoding mitochondria-anchoring protein syntaphilin, improved mitochondrial motility in axons after in vivo sciatic nerve injury and enhanced axonal regeneration [59]. Similarly, overexpression of mammalian-specific mitochondrial protein Armcx1in adult retinal ganglion cells mobilized mitochondria in axons and promoted neuronal survival as well as axonal re-growth [60].

These studies raise a possibility that higher mitochondria number and motility in injured neurons may provide better regenerative capacity both in the peripheral nervous system and the central nervous system (CNS) (Fig. 1c) [61-63].

\section{Mitochondrial therapy}

The concept of "mitochondrial medicine", which refers to medical intervention targeting mitochondria, boots a new line of biomedical endeavor. Mitochondrial therapy aims to restore mitochondrial functions, such as mildly inducing mitochondrial uncoupling, boosting energy production, and antagonizing the release of ROS. New drugs in forms of mitochondrial membrane uncoupling agents (eg. 2,4-dinitrophenel, uncoupling protein-2, uncoupling protein-3), electron transfer chain-boosting substrates (eg. dichloroacetate, thiamine), metabolism modulators (eg. Metforin) and antioxidants (eg. coenzyme $\mathrm{Q}_{10}$, MitoQ, RP103) have been developed or preclinically tested $[2,64,65]$. By the end of July 2018 , there were more than 400 completed or ongoing clinical trials for mitochondria-targeted medical intervention registered at ClinicalTrials.gov. However, there is currently no medicine to cure mitochondria-related diseases caused by inefficient energy production, and the loss of normal physiological ROS function. Therefore, a new paradigm of mitochondrial therapy based on organelle delivery strategy was established. Supplement of healthy mitochondria into cells containing damaged mitochondria was beneficial to improve energy generation, reverse excessive ROS production, and restore mitochondrial function. Findings in recent years 


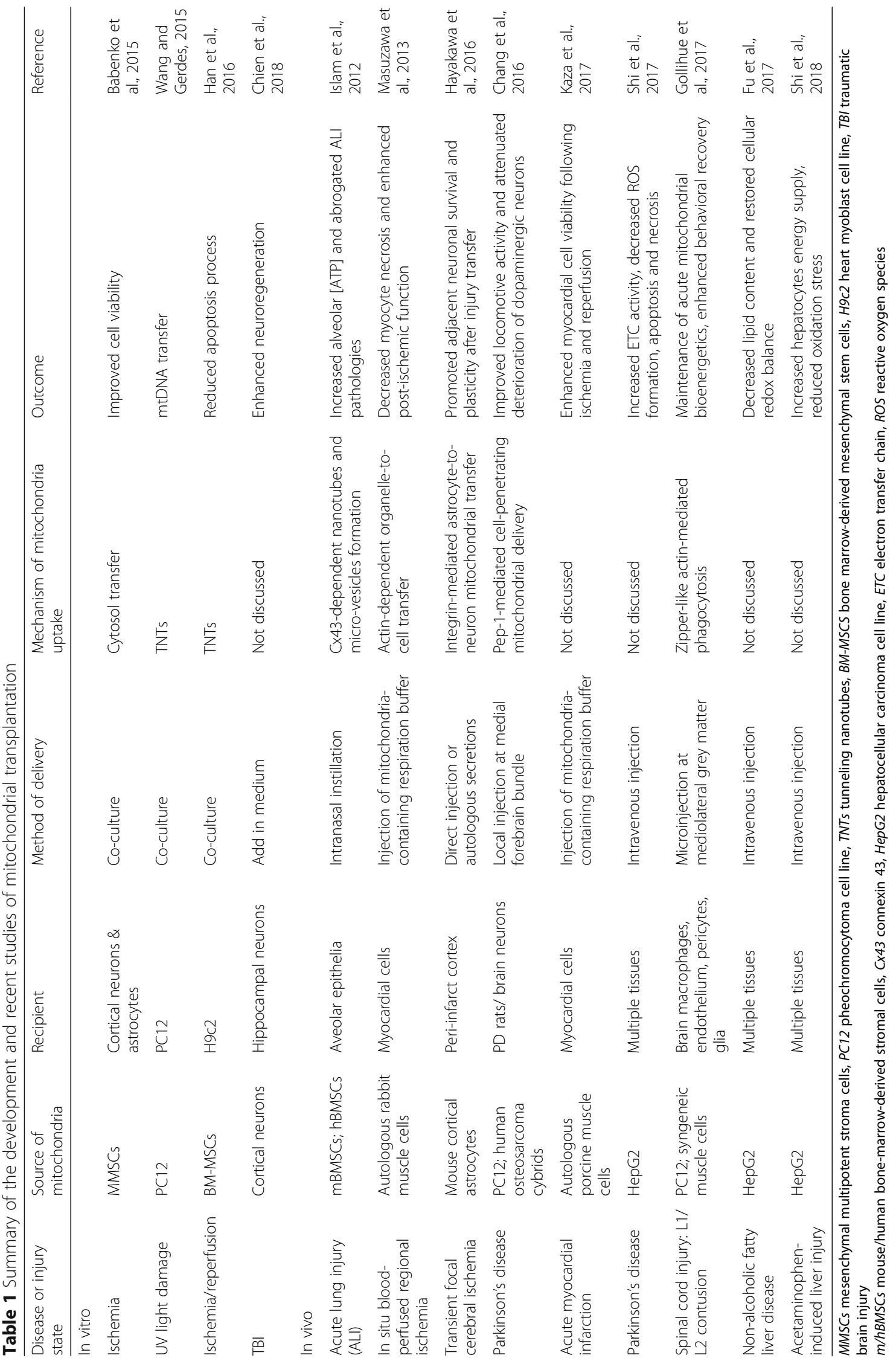


Table 2 Registered interventional studies for mitochondrial transplantation on ClinicalTrials.gov

\begin{tabular}{|c|c|c|c|c|c|}
\hline Conditions/Diseases & Status & Phase & Intervention & Mitochondria donor & NCT number \\
\hline $\begin{array}{l}\text { Age-related deterioration of } \\
\text { oocyte quality }\end{array}$ & Withdrawn & $1 \& 2$ & Injection of autologous mitochondria to the oocytes & $\begin{array}{l}\text { Autologous granulosa } \\
\text { cells }\end{array}$ & NCT01631578 \\
\hline Infertility & Completed & NA & $\begin{array}{l}\text { Autologous micro-injection of mitochondria into the } \\
\text { oocytes during ICSI }\end{array}$ & $\begin{array}{l}\text { Autologous ovarian stem } \\
\text { cells }\end{array}$ & NCT02586298 \\
\hline $\begin{array}{l}\text { Mitochondrial diseases: } \\
\text { Pearson Syndrome }\end{array}$ & $\begin{array}{l}\text { Not yet } \\
\text { recruiting }\end{array}$ & $\begin{array}{l}\text { Early } \\
1\end{array}$ & $\begin{array}{l}\text { Mitochondria augmentation therapy: transplantation of } \\
\text { autologous stem cell enriched with MNV-BLD }\end{array}$ & $\begin{array}{l}\text { Autologous peripheral } \\
\text { hematopoietic stem cells }\end{array}$ & NCT03384420 \\
\hline $\begin{array}{l}\text { Extracorporeal membrane } \\
\text { oxygenation complication }\end{array}$ & Recruiting & NA & $\begin{array}{l}\text { Autologous mitochondria injected or infused into the } \\
\text { ischemic myocardium }\end{array}$ & $\begin{array}{l}\text { Autologous skeletal } \\
\text { muscle cells }\end{array}$ & NCT02851758 \\
\hline
\end{tabular}

NA not applicable, ICSI intracytoplasmic sperm injection, ${ }^{a}$ MNV-BLD refers to blood-derived mitochondria

have demonstrated the promising outcome upon receiving mitochondrial delivery using in vitro and in vivo models (Table 1) and in several completed or on-going clinical trials (Table 2) $[2,66]$. In the following section, we will review recent application of mitochondrial delivery techniques in experimental animals modelling human diseases and highlight the therapeutic potential of delivering isolated mitochondria for the management of neurodegenerative diseases, cerebral stroke, and TBI.

\section{Mechanism of mitochondrial uptake by cells}

Mechanisms underlying mitochondrial internalization have been reported (Table 1 and Fig. 2) [67]. Organelle transfer through cell-to-cell fusion or via mitochondriacontaining vesicles was observed in bone-marrow-derived stroma cell-to-lung epithelium mitochondrial transfer to mitigate acute lung injury [68]. Tunneling nanotubes (TNTs)-dependent mitochondrial transfer has been wellcharacterized [68-70]. This actin-based structure was found to mediate mitochondrial exchange between healthy and UV stress-damaged PC12 cells to prevent damaged cells from apoptosis. Nanotube-mediated mitochondrial transfer from co-cultured mesenchymal stem cells to epithelium was reported to rescue cigarette smoke-induced lung damage [71]. Notably, recent study discovered an intriguing mechanism by which strokeinduced activated astrocytes released mitochondriacontaining particles and these particles entered damaged neurons through actin-dependent endocytosis to prevent neuronal death $[72,73]$.

\section{Mitochondrial delivery for neurodegenerative diseases, cerebral stroke and TBI}

As in vivo mitochondrial supplementation in cardiac ischemia models set a milestone for organelle deliverybased therapy, this approach was also applied to neurodegenerative diseases, cerebral stroke, and TBI. Hereinafter, we review the approach of mitochondrial delivery in degenerating, hypoxemic, or injured nervous system.

\section{Neurodegenerative diseases}

Due to limited understanding of molecular basis underlying AD pathogenesis, available drugs approved by the Food and Drug Administration of the United States for AD, such as acetylcholinesterase inhibitors galantamine, donepezil, and rivastigmine, can simply relieve the symptoms [74, 75]. Since the 1980s, many studies have revealed mitochondrial abnormalities in the $\mathrm{AD}$ subjects, including structural change, deficiency of Kreb cycles enzymes, reduced cytochrome oxidase activity, and the disturbance of calcium homeostasis [76-79]. Mitochondrial delivery in AD model was originally conducted in the in vitro cybrid cell system. Cybrids were generated by fusing mtDNA-depleted human neuroblastoma cell line, SH-SY5Y, or teratocarcinoma cells Ntera2/D1 (NT2), with mitochondria from platelets of AD patients $[80,81]$. Reduced activity of mitochondrial complex IV, elevated ROS production, higher cytosolic calcium concentration, and defective cytochrome c oxidase, were found in the $\mathrm{AD}$ cybrids compared to non- $\mathrm{AD}$ control cybrids. Based on these discoveries, mitochondrial cascade hypothesis in the pathogenesis of sporadic $A D$ was then proposed by Khan et al, suggesting that baseline mitochondrial function and durability determine aging-related mitochondrial changes and would progress to $\mathrm{AD}[82,83]$. Although pre-clinical studies on many anti-oxidants, such as $\alpha$-tocopherol, for treating $\mathrm{AD}$ were found effective in experimental $\mathrm{AD}$ animal models, few clinical trials have succeeded. Given the complexity of AD pathophysiology as well as limited efficiency of drug delivery, improved therapeutic strategy of mitochondrial therapy is needed.

Mitochondrial dysfunction aggravates the progression of PD, manifested by increased oxidative stress, dysregulated bioenergetic homeostasis, and reduced viability of affected SN dopaminergic neurons. While mitochondriatargeting antioxidant was considered of great potential for treating PD, existing agents have limited effect on preventing $\mathrm{PD}$ from deterioration even if there was promising outcome in animal models and pre-clinical tests $[84,85]$. For example, antioxidant drugs, coenzyme Q10 and creatine monohydrate, failed to significantly alleviate the progression in patients with PD in the clinical 


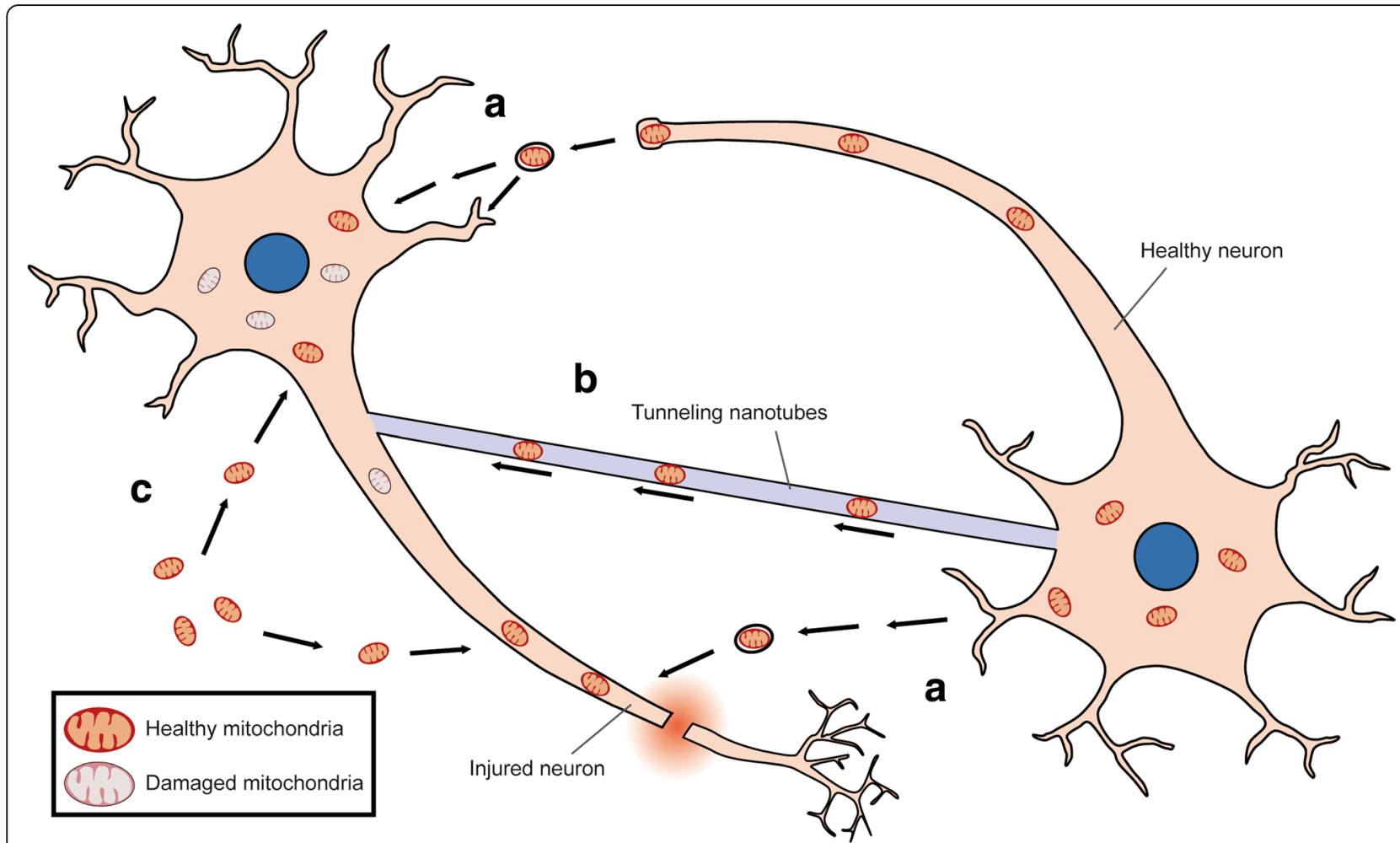

Fig. 2 Mechanisms underlying mitochondria internalization. Three uptake routes for mitochondrial therapy: a Mitochondria-containing vesicles are released from healthy neurons (or donor cells) and then internalized into injured neurons. $\mathbf{b}$ Healthy mitochondria are transported via the actin-based tunneling nanotubes between donor cells and injured neurons. $\mathbf{c}$ Extracellular healthy mitochondria through focal administration are internalized into injured neuron

trials $[86,87]$. Therefore, instead of targeting a single specific aspect of mitochondrial function, supplementing healthy mitochondria to damaged regions in PD brain may potentially be an innovative strategy for improving clinical outcome. To this end, several studies set out to examine the efficacy and feasibility of mitochondrial delivery in inhibiting PD progression. Chang et al. demonstrated that cell-penetrating peptide-based mitochondrial delivery in 6-hydroxydopamine (OHDA)-treated PC12 cells rescued mitochondrial respiratory function, improved cell viability, and promoted neurite growth when treated the PC12 cells with nerve growth factor [88]. Xenogeneic/ allogeneic injection of mitochondria into medical forebrain bundle (MFB) of 6-OHDA-unilaterally infused PD rats enhanced the survival of dopaminergic neurons as well as effectively sustained mitochondrial functions by restoring the normal level of mitochondrial complex I-IV and relieving mitochondrial oxidative stress in vivo. Upon receiving supplemented mitochondria, protein levels involved in mitochondrial fusion (Mfn2, OPA1), fission (Drp1), and deterioration (Parkin) in dopaminergic neurons within SN were restored. In addition, mitochondrial transplantation in MFB improved locomotive activity of 6OHDA-induced PD rat. In the other study conducted by Shi et al., MPP (1-methyl-4-phenyl-pyridinium)-treated
SH-SY5Y cells incubated with intact isolated mitochondria improved cell viability in a dose-dependent manner [89]. ATP production, mitochondrial complex I activity and cell survival were rescued after mitochondrial supplementation while the level of ROS significantly lowered, compared to $\mathrm{MPP}^{+}$control cells. The initial report by Shi et al. showed that systemic intravenous mitochondrial administration to respiratory chain inhibitor MPTP (1-methyl-4phenyl-1,2,3,6-tetrahydropyridine)-induced PD mouse model prevented PD progression [89]. In vivo distribution of intravenously-injected mitochondria was found in multiple organs, including brain, $2 \mathrm{~h}$ after intravenous injection. As a result, striatal mitochondria in MPTP-induced PD mice showed increased ATP content, restored mitochondrial complex I activity, and decreased ROS production with improved locomotor activity.

\section{Stroke}

Current intervention for stroke is limited owing to narrow therapeutic time window after the occurrence of ischemic stroke. Ischemia-induced OGD in affected regions leads to low ATP production, excessive ROS release from mitochondria, ionic disequilibrium across mitochondrial membranes, and eventually programmed cell death $[17,90]$. As accumulating evidence links mitochondrial deficit to brain 
impairment following ischemic stroke, therapeutic regimen was developed aiming to restore mitochondrial physiology. In light of new concept of intercellular organelle-transfer, Hayakswa et al. demonstrated that CD38 signalling mediated release of functional mitochondria from activated astrocyte. These mitochondria then entered damaged cortical neurons, restored ATP level and neuronal viability after OGD injury. Treatment with extracellular mitochondria-containing particles, released from cultured astrocytes in a mouse model of focal cerebral ischaemia, provided neuroprotection. In vitro astrocyte-to-neuron mitochondrial delivery and in vivo astrocyte-derived mitochondrial transfer promoted neuronal survival, plasticity, as well as improved behavior outcome [72]. Besides, it has been reported that mitochondria are transferred from mesenchymal multipotent stromal cells to co-cultured neurons. Intravenous administration of mesenchymal multipotent stromal cells to MCAO rats reduced infarction area and improved post-stroke neurological indexes. Treatment of "primed" stem cells, which had been previously co-cultured with neuron cells, caused a more pronounced beneficial outcome in rats after stroke [73]. Transfer of exogenous mitochondria via local intracerebral or systemic intraarterial injection reduced brain lesion, cell death, and restored motor function in MCAO rats [91]. In addition, autologous mitochondrial transplantation has been studied in rabbit ischemic heart model. After regional ischemia, autologous skeletal muscle-derived mitochondria were injected into ischemic zone of heart prior to reperfusion. Mitochondrial transplantation significantly reduced myocyte necrosis, infarction volume and improved post-ischemic recovery of cardiac function without eliciting any immune or inflammatory response. Moreover, biochemical markers of myocardial infarction, creatine kinase-muscle/brain isoenzyme and cardiac troponin I, were reduced after mitochondrial transplantation [92]. Follow-up study using porcine cardiac ischemia/reperfusion model showed similar results in that autologous mitochondrial transplantation enhanced post-ischemic myocardial cell viability, reduced infarction size and deceased myocardial injury biomarkers [93]. These successful cases highlight the effective mitochondrial therapy in post-stroke neuroprotection, preserving cell viability and promoting functional recovery.

\section{Traumatic brain injury}

Traumatic injury in the CNS, including spinal cord injury (SCI) and TBI, has been one of the most pressing medical issues worldwide according to its high incidence and lack of effective treatment strategy. The initial study investigating the feasibility of mitochondrial transplantation in SCI reported that supplementation of a pool of healthy mitochondria into L1/L2 contusion SCI rat model acutely sustained cellular bioenergetics in injured spinal cord and improved locomotor activity, whereas long-term effect on neuroprotection and tissue sparing were not observed [94]. On the other aspect, TBI is highly regarded as a global healthcare issue given that it has been the leading cause of injury death according to Center for Disease Control and Prevention, USA [95]. By the end of April in 2018, approximately 69 million of individuals annually suffer from TBI [96]. Post-traumatic mitochondrial deficit includes alternation of membrane structure and calcium homeostasis, uncoupled electron transfer system, accumulation of ROS and induction of apoptosis [97, 98]. Such structural damage and metabolic/physiological dysfunction of mitochondria dampen neuronal viability and plasticity. Disruption of mitochondrial dynamics has also been implicated in TBI-induced behavior impairment and the loss of cognitive function [16, 99]. Accumulating data suggest that mitochondrial therapy could be beneficial for clinical TBI treatment yet the efficacy of mitochondrial transplantation for treating TBI had not been evaluated. A recent report by our laboratory revealed increased mitochondrial fission hours after injury in hippocampal neurons. While retrograde transportation of mitochondria from injury site to cell body was observed in the injured neurites, mitochondria were transported toward newly formed growth cones in re-growing axons. Supplement of freshly isolated mitochondria derived from rat cortical neurons to injured hippocampal neurons promoted neurite re-growth and restored membrane potential of injured neurons [13]. As these findings point to a pivotal role of mitochondrial function in modulating TBI pathophysiology, mitochondrial transplantation could well be a novel strategy for clinical treatment of TBI.

\section{Clinical application of mitochondrial transplantation Techniques for mitochondrial delivery}

The effectiveness of mitochondrial therapy is expected to be variable among patients due to the heterogeneity of pathogenesis and efficiency of mitochondrial internalization into the affected tissues. Successful uptake of mitochondria by target tissues depends on the amount, quality of mitochondria and proper routes of organelle delivery. Therefore, better understanding of the mechanisms underlying mitochondrial delivery and cellular uptake will facilitate the translation of mitochondrial transplantation in clinic.

A number of in vivo studies documented feasible approaches of mitochondrial transplantation, including microinjection directly to affected sites in SCI, stroke, and PD models [88, 92-94], and intravenous administration in PD and fatty liver models $[89,100]$. In PD, to improve functional incorporation of supplemented mitochondria, a novel strategy of peptide-mediated allogenic mitochondrial delivery (PMD) was applied to neurotoxin-induced PD rats. Direct microinjection of Pep-1-modified allogenic mitochondria into MFB promoted cellular uptake of 
mitochondria compared to the injection of naïve mitochondria or xenogenic PMD. It was clear that PMD successfully rescued impaired mitochondrial respiration, attenuated oxidative damage, sustained neuron survival, and restored locomotor activity of PD rats [88]. Nevertheless, the conjugation ratio of Pep-1 and mitochondria should be optimized to avoid undesired mitochondrial aggregation. Moreover, the conjugation time and human manipulation should be minimized before clinical translation. Another study systemically administered isolated mitochondria via tail vein improved locomotor activity in PD mouse model, albeit differential distribution of injected mitochondria in brain, heart, liver, kidney, and muscle [89]. The feasibility of intravenous mitochondrial delivery was achieved by smaller size of the organelle $(\sim$ $1 \mu \mathrm{m}$ in diameter) compared to that of red blood cells (6 $\sim \mu \mathrm{m}$ in diameter) and that supplemented mitochondria are not to be incorporated into red blood cells to interfere oxygen transport.

\section{Clinical trials}

The burgeoning of mitochondrial therapy opened a new era for reversing mitochondria function in human diseases. Thus far, few registered clinical trials for treating neurodegenerative diseases, stroke, or TBI based on mitochondrial delivery technique have been launched. To date, there is only one completed trial which aimed to treat infertility by autologous mitochondrial injection into oocytes (Table 2, NCT\#02586298). Autologous ovarian mitochondria were isolated prior to in vitro intracytoplasmic sperm injection (ICSI). The outcome was determined by ongoing rate of pregnancy within 12 weeks after mitochondrial therapy, as the improvement in preimplantation genetic screening and embryo quality were also evaluated. An ongoing trial tries to demonstrate the feasibility of mitochondrial transplantation, using autologous mitochondrial injection (Table 2, NCT\#02851758), for rehabilitating myocardial ischemia/reperfusion injury and is currently recruiting participants. Mitochondria will be isolated from autologous skeletal muscle from patients undergoing surgical re-operation or catheterization and directly injected into affected myocardium or proximal aorta, or via intracoronary infusion. The outcome will be measured by the safety and the improvement of ventricular function after therapeutic intervention.

\section{Conclusions}

Previous proposals for treating mitochondrial dysfunction have been targeting specific mitochondrial residents and fusion/fission regulators [64, 65]. The outcome of these approaches has not been satisfactory. The emerging line of approach is to supplement freshly isolated mitochondria (mitochondrial transplantation) to injury sites. Alternatively, in the case of stroke, to activate astrocyte for releasing mitochondria-containing particles for intercellular transfer of mitochondria (to neurons). Our previous work showed that supplement of freshly isolated mitochondria promoted neurite re-growth and restored the membrane potential of injured hippocampal neurons [13]. Nonetheless, it is conceivable that clinical translation of mitochondrial delivery on TBI would face great challenge. For instance, checkpoint at the blood brain barrier should be considered to improve the effectiveness and the volume used would also be a limiting factor. The therapeutic outcome of mitochondrial transplantation largely depends upon the isolation protocol, quality of isolated mitochondria, and tissue-specific differential uptake. Biocompatible materials for packaging mitochondria may facilitate the delivery and the subsequent uptake by cells. For clinical application, it is more feasible to isolate mitochondria from peripheral tissues to obtain sufficient amount of allogenic mitochondria for the treatment of CNS diseases. Based on our experience, the percentage of functional mitochondria after isolation and the quality maintenance over time are crucial measurement for the success of promoting neuronal regeneration. While published data showed that peptide-based allogeneic mitochondrial delivery successfully entered target cells and recovered damaged tissues without triggering significant immune response in PD model, the efficacy of PMD in cerebral stroke and TBI patients has yet to be determined [88]. More importantly, regenerative outcome characterized by neurite re-growth, de novo synaptogenesis, and the restoration of neuronal activity should be inclusively evaluated in addition to the maintenance of cell survival. Thus, future efforts on the feasibility and efficacy of allogeneic mitochondrial delivery on treating a wide range of mitochondria-related diseases will expedite the clinical translation.

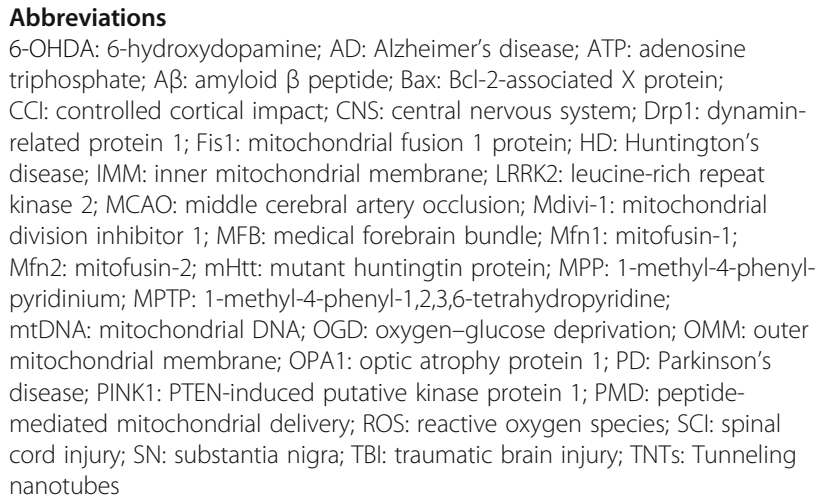

\section{Acknowledgements}

Not applicable.

\section{Authors' contributions}

LC and CYC conceived and designed the content of the paper. CYC and MZL contributed to literature search and Table preparation. MZL contributed 
to figures preparation. LC, CYC, and MZL wrote the manuscript. All authors read and approved the final manuscript.

\section{Funding}

This work was supported by funding from the National Health Research Institutes, Taiwan (Grant numbers: NHRI-EX106-10206NI; NHRI-EX108$10813 \mathrm{NI})$, to Linyi Chen.

\section{Availability of data and materials}

Not applicable.

\section{Ethics approval and consent to participate}

Not applicable.

\section{Consent for publication}

Not applicable.

\section{Competing interests}

The authors declare that they have no competing interests.

Received: 20 December 2018 Accepted: 24 May 2019 Published online: 14 June 2019

\section{References}

1. Tait SW, Green DR. Mitochondria and cell signalling. J Cell Sci. 2012;125: 807-15.

2. Gollihue JL, Rabchevsky AG. Prospects for therapeutic mitochondrial transplantation. Mitochondrion. 2017;35:70-9.

3. Rahman J, Rahman S. Mitochondrial medicine in the omics era. Lancet. 2018;391:2560-74.

4. Friedman JR, Nunnari J. Mitochondrial form and function. Nature. 2014;505: 335-43.

5. Westermann B. Mitochondrial fusion and fission in cell life and death. Nat Rev Mol Cell Biol. 2010;11:872-84.

6. Sedlackova L, Korolchuk VI. Mitochondrial quality control as a key determinant of cell survival. Biochim Biophys Acta, Mol Cell Res. 1866; 2019:575-87.

7. Friedman JR, Lackner LL, West M, DiBenedetto JR, Nunnari J, Voeltz GK. ER tubules mark sites of mitochondrial division. Science. 2011;334:358.

8. Suarez-Rivero JM, Villanueva-Paz M, de la Cruz-Ojeda P, de la Mata M, Cotan D, Oropesa-Avila M, de Lavera I, Alvarez-Cordoba M, Luzon-Hidalgo R, Sanchez-Alcazar JA. Mitochondrial dynamics in mitochondrial diseases. Diseases. 2016;5

9. Mishra P. Interfaces between mitochondrial dynamics and disease. Cell Calcium. 2016;60:190-8.

10. Chan DC. Mitochondria: dynamic organelles in disease, aging, and development. Cell. 2006;125:1241-52.

11. Li Z, Okamoto K, Hayashi Y, Sheng M. The importance of dendritic mitochondria in the morphogenesis and plasticity of spines and synapses. Cell. 2004;119:873-87.

12. Chang DT, Reynolds IJ. Differences in mitochondrial movement and morphology in young and mature primary cortical neurons in culture. Neuroscience. 2006;141:727-36.

13. Chien L, Liang MZ, Chang CY, Wang C, Chen L. Mitochondrial therapy promotes regeneration of injured hippocampal neurons. Biochim Biophys Acta. 2018;1864:3001-12.

14. Kann O, Kovacs R. Mitochondria and neuronal activity. Am J Phys Cell Phys. 2007;292:C641-57.

15. Gao J, Wang L, Liu J, Xie F, Su B, Wang X. Abnormalities of Mitochondrial Dynamics in Neurodegenerative Diseases. Antioxidants (Basel). 2017;6:2.

16. Fischer TD, Hylin MJ, Zhao J, Moore AN, Waxham MN, Dash PK. Altered mitochondrial dynamics and TBI pathophysiology. Front Syst Neurosci. 2016;10:29.

17. Yang JL, Mukda S, Chen SD. Diverse roles of mitochondria in ischemic stroke. Redox Biol. 2018;16:263-75.

18. Schon EA, Przedborski S. Mitochondria: the next (neurode)generation. Neuron. 2011;70:1033-53.

19. Swerdlow RH. Mitochondria and mitochondrial cascades in Alzheimer's disease. J Alzheimers Dis. 2018;62:1403-16.
20. Cho DH, Nakamura T, Fang J, Cieplak P, Godzik A, Gu Z, Lipton SA. Snitrosylation of Drp1 mediates beta-amyloid-related mitochondrial fission and neuronal injury. Science. 2009;324:102-5.

21. Bossy B, Petrilli A, Klinglmayr E, Chen J, Lutz-Meindl U, Knott AB, Masliah E, Schwarzenbacher R, Bossy-Wetzel E. S-Nitrosylation of DRP1 does not affect enzymatic activity and is not specific to Alzheimer's disease. J Alzheimers Dis. 2010;20(Suppl 2):S513-26.

22. Jakob-Roetne R, Jacobsen H. Alzheimer's disease: from pathology to therapeutic approaches. Angew Chem Int Ed Eng. 2009;48:3030-59.

23. Cummings JL. Alzheimer's disease. N Engl J Med. 2004;351:56-67.

24. Manczak M, Kandimalla R, Fry D, Sesaki H, Reddy PH. Protective effects of reduced dynamin-related protein 1 against amyloid beta-induced mitochondrial dysfunction and synaptic damage in Alzheimer's disease. Hum Mol Genet. 2016;25:5148-66.

25. Baek SH, Park SJ, Jeong JI, Kim SH, Han J, Kyung JW, Baik SH, Choi Y, Choi BY, Park JS, et al. Inhibition of Drp1 ameliorates synaptic depression, Abeta deposition, and cognitive impairment in an Alzheimer's disease model. J Neurosci. 2017;37:5099-110.

26. Martin-Maestro P, Gargini R, Garcia E, Perry G, Avila J, Garcia-Escudero V. Slower dynamics and aged mitochondria in sporadic Alzheimer's disease. Oxidative Med Cell Longev. 2017:2017:9302761.

27. Wilhelmus MM, van der Pol SM, Jansen $Q$, Witte ME, van der Valk $P$, Rozemuller AJ, Drukarch B, de Vries HE, Van Horssen J. Association of Parkinson disease-related protein PINK1 with Alzheimer disease and multiple sclerosis brain lesions. Free Radic Biol Med. 2011;50:469-76.

28. Du F, Yu Q, Yan S, Hu G, Lue LF, Walker DG, Wu L, Yan SF, Tieu K, Yan SS. PINK1 signalling rescues amyloid pathology and mitochondrial dysfunction in Alzheimer's disease. Brain. 2017:140:3233-51.

29. Shirendeb UP, Calkins MJ, Manczak M, Anekonda V, Dufour B, McBride JL, Mao P, Reddy PH. Mutant huntingtin's interaction with mitochondrial protein Drp1 impairs mitochondrial biogenesis and causes defective axonal transport and synaptic degeneration in Huntington's disease. Hum Mol Genet. 2012;21:406-20

30. Wang X, Yan MH, Fujioka H, Liu J, Wilson-Delfosse A, Chen SG, Perry G, Casadesus G, Zhu X. LRRK2 regulates mitochondrial dynamics and function through direct interaction with DLP1. Hum Mol Genet. 2012;21:1931-44.

31. Plotegher N, Duchen MR. Crosstalk between lysosomes and mitochondria in Parkinson's disease. Front Cell Dev Biol. 2017:5:110.

32. Reeve AK, Grady JP, Cosgrave EM, Bennison E, Chen C, Hepplewhite PD, Morris CM. Mitochondrial dysfunction within the synapses of substantia nigra neurons in Parkinson's disease. NPJ Parkinsons Dis. 2018;4:9.

33. Quintanilla RA, Johnson GV. Role of mitochondrial dysfunction in the pathogenesis of Huntington's disease. Brain Res Bull. 2009;80:242-7.

34. Carmo C, Naia L, Lopes C, Rego AC. Mitochondrial dysfunction in Huntington's disease. Adv Exp Med Biol. 2018;1049:59-83.

35. Song W, Chen J, Petrilli A, Liot G, Klinglmayr E, Zhou Y, Poquiz P, Tjong J, Pouladi MA, Hayden MR, et al. Mutant huntingtin binds the mitochondrial fission GTPase dynamin-related protein-1 and increases its enzymatic activity. Nat Med. 2011;17:377-82.

36. Choo YS, Johnson GV, MacDonald M, Detloff PJ, Lesort M. Mutant huntingtin directly increases susceptibility of mitochondria to the calciuminduced permeability transition and cytochrome c release. Hum Mol Genet. 2004:13:1407-20.

37. Panov AV, Gutekunst CA, Leavitt BR, Hayden MR, Burke JR, Strittmatter WJ, Greenamyre JT. Early mitochondrial calcium defects in Huntington's disease are a direct effect of polyglutamines. Nat Neurosci. 2002;5:731-6.

38. Milakovic T, Quintanilla RA, Johnson GV. Mutant huntingtin expression induces mitochondrial calcium handling defects in clonal striatal cells: functional consequences. J Biol Chem. 2006;281:34785-95.

39. Kim J, Moody JP, Edgerly CK, Bordiuk OL, Cormier K, Smith K, Beal MF, Ferrante RJ. Mitochondrial loss, dysfunction and altered dynamics in Huntington's disease. Hum Mol Genet. 2010;19:3919-35.

40. Ehrnhoefer DE, Martin DDO, Schmidt ME, Qiu X, Ladha S, Caron NS, Skotte $\mathrm{NH}$, Nguyen YTN, Vaid $\mathrm{K}$, Southwell AL, et al. Preventing mutant huntingtin proteolysis and intermittent fasting promote autophagy in models of Huntington disease. Acta Neuropathol Commun. 2018;6:16

41. Wong YC, Holzbaur EL. The regulation of autophagosome dynamics by huntingtin and HAP1 is disrupted by expression of mutant huntingtin, leading to defective cargo degradation. J Neurosci. 2014;34:1293-305.

42. Seong IS, Ivanova E, Lee JM, Choo YS, Fossale E, Anderson M, Gusella JF, Laramie JM, Myers RH, Lesort M, MacDonald ME. HD CAG repeat implicates 
a dominant property of huntingtin in mitochondrial energy metabolism Hum Mol Genet. 2005;14:2871-80.

43. Beal MF, Brouillet E, Jenkins BG, Ferrante RJ, Kowall NW, Miller JM, Storey E, Srivastava R, Rosen BR, Hyman BT. Neurochemical and histologic characterization of striatal excitotoxic lesions produced by the mitochondrial toxin 3-nitropropionic acid. J Neurosci. 1993;13:4181-92.

44. Brouillet E, Hantraye P, Ferrante RJ, Dolan R, Leroy-Willig A, Kowall NW, Beal MF. Chronic mitochondrial energy impairment produces selective striatal degeneration and abnormal choreiform movements in primates. Proc Natl Acad Sci U S A. 1995;92:7105-9.

45. Barsoum MJ, Yuan H, Gerencser AA, Liot G, Kushnareva Y, Graber S, Kovacs I, Lee WD, Waggoner J, Cui J, et al. Nitric oxide-induced mitochondrial fission is regulated by dynamin-related GTPases in neurons. EMBO J. 2006;25:3900-11.

46. Grohm J, Kim SW, Mamrak U, Tobaben S, Cassidy-Stone A, Nunnari J, Plesnila N, Culmsee C. Inhibition of Drp1 provides neuroprotection in vitro and in vivo. Cell Death Differ. 2012;19:1446.

47. Zhao Y, Chen F, Chen S, Liu X, Cui M, Dong Q. The Parkinson's diseaseassociated gene PINK1 protects neurons from ischemic damage by decreasing mitochondrial translocation of the fission promoter Drp1. J Neurochem. 2013;127:711-22.

48. Peng C, Rao W, Zhang L, Wang K, Hui H, Wang L, Su N, Luo P, Hao Y-L, Tu Y, et al. Mitofusin 2 ameliorates hypoxia-induced apoptosis via mitochondrial function and signaling pathways. Int J Biochem Cell Biol. 2015;69:29-40.

49. Hartmann JF. Electron microscopy of motor nerve cells following section of axones. Anat Rec. 1954;118:19-33.

50. Hudson G, Lazarow A, Hartmann JF. A quantitative electron microscopic study of mitochondria in motor neurones following axonal section. Exp Cell Res. 1961;24:440-56.

51. Lentz TL. Fine structure of sensory ganglion cells during limb regeneration of the newt Triturus. J Comp Neurol. 1967;131:301-22.

52. Smith KRJ. The fine structure of neurons of dorsal root ganglia after stimulating or cutting the sciatic nerve. J Comp Neurol. 1961;116:103-15.

53. Dimova RN, Markov DV. Changes in the mitochondria in the initial part of the axon during regeneration. Acta Neuropathol. 1976;36:235-42.

54. Niu F, Dong J, Xu X, Zhang B, Liu B. Mitochondrial division inhibitor 1 prevents early-stage induction of Mitophagy and accelerated cell death in a rat model of moderate controlled cortical impact brain injury. World Neurosurg. 2019;122:e1090-e101.

55. Di Pietro V, Lazzarino G, Amorini AM, Signoretti S, Hill LJ, Porto E, Tavazzi B, Lazzarino G, Belli A. Fusion or fission: the Destiny of mitochondria in traumatic brain injury of different severities. Sci Rep. 2017;7:9189.

56. Chien L, Chen WK, Liu ST, Chang CR, Kao MC, Chen KW, Chiu SC, Hsu ML, Hsiang IC, Chen YJ, Chen L. Low-dose ionizing radiation induces mitochondrial fusion and increases expression of mitochondrial complexes I and III in hippocampal neurons. Oncotarget. 2015;6:30628-39.

57. Xu Y, Chen M, Hu B, Huang R, Hu B. In vivo imaging of mitochondrial transport in single-axon regeneration of zebrafish Mauthner cells. Front Cell Neurosci. 2017;11:4.

58. Han SM, Baig HS, Hammarlund M. Mitochondria localize to injured axons to support regeneration. Neuron. 2016;92:1308-23.

59. Zhou B, Yu P, Lin MY, Sun T, Chen Y, Sheng ZH. Facilitation of axon regeneration by enhancing mitochondrial transport and rescuing energy deficits. J Cell Biol. 2016;214:103-19.

60. Cartoni R, Norsworthy MW, Bei F, Wang C, Li S, Zhang Y, Gabel CV, Schwarz $T L, H e Z$. The mammalian-specific protein Armcx 1 regulates mitochondrial transport during axon regeneration. Neuron. 2016;92:1294-307.

61. Patron LA, Zinsmaier KE. Mitochondria on the road to power axonal regeneration. Neuron. 2016;92:1152-4.

62. Cartoni R, Pekkurnaz G, Wang C, Schwarz TL, He Z. A high mitochondrial transport rate characterizes CNS neurons with high axonal regeneration capacity. PLoS One. 2017;12:e0184672.

63. Smith GM, Gallo G. The role of mitochondria in axon development and regeneration. Dev Neurobiol. 2018;78:221-37.

64. Wang W, Karamanlidis G, Tian R. Novel targets for mitochondrial medicine. Sci Transl Med. 2016;8:326rv3.

65. El-Hattab AW, Zarante AM, Almannai M, Scaglia F. Therapies for mitochondrial diseases and current clinical trials. Mol Genet Metab. 2017;122:1-9.

66. Liu CS, Chang JC, Kuo SJ, Liu KH, Lin TT, Cheng WL, Chuang SF. Delivering healthy mitochondria for the therapy of mitochondrial diseases and beyond. Int J Biochem Cell Biol. 2014;53:141-6.
67. Caicedo A, Aponte PM, Cabrera F, Hidalgo C, Khoury M. Artificial mitochondria transfer: current challenges, advances, and future applications. Stem Cells Int. 2017;2017:7610414.

68. Islam MN, Das SR, Emin MT, Wei M, Sun L, Westphalen K, Rowlands DJ, Quadri SK, Bhattacharya S, Bhattacharya J. Mitochondrial transfer from bonemarrow-derived stromal cells to pulmonary alveoli protects against acute lung injury. Nat Med. 2012;18:759-65.

69. Wang $\mathrm{X}$, Gerdes HH. Transfer of mitochondria via tunneling nanotubes rescues apoptotic PC12 cells. Cell Death Differ. 2015;22:1181-91.

70. Han H, Hu J, Yan Q, Zhu J, Zhu Z, Chen Y, Sun J, Zhang R. Bone marrowderived mesenchymal stem cells rescue injured H9c2 cells via transferring intact mitochondria through tunneling nanotubes in an in vitro simulated ischemia/reperfusion model. Mol Med Rep. 2016;13:1517-24.

71. Li X, Zhang Y, Yeung SC, Liang Y, Liang X, Ding Y, Ip MS, Tse HF, Mak JC, Lian Q. Mitochondrial transfer of induced pluripotent stem cell-derived mesenchymal stem cells to airway epithelial cells attenuates cigarette smoke-induced damage. Am J Respir Cell Mol Biol. 2014;51:455-65.

72. Hayakawa K, Esposito E, Wang X, Terasaki Y, Liu Y, Xing C, Ji X, Lo EH. Transfer of mitochondria from astrocytes to neurons after stroke. Nature. 2016;535:551-5.

73. Babenko VA, Silachev DN, Zorova LD, Pevzner IB, Khutornenko AA, Plotnikov EY, Sukhikh GT, Zorov DB. Improving the post-stroke therapeutic potency of mesenchymal multipotent stromal cells by Cocultivation with cortical neurons: the role of crosstalk between cells. Stem Cells Transl Med. 2015;4: $1011-20$.

74. Kim SH, Kandiah N, Hsu JL, Suthisisang C, Udommongkol C, Dash A. Beyond symptomatic effects: potential of donepezil as a neuroprotective agent and disease modifier in Alzheimer's disease. Br J Pharmacol. 2017;174:4224-32.

75. Jan AT, Azam M, Rahman S, Almigeiti AMS, Choi DH, Lee EJ, Haq QMR, Choi I. Perspective insights into disease progression, diagnostics, and therapeutic approaches in Alzheimer's disease: a judicious update. Front Aging Neurosci. 2017;9:356

76. Hirai K, Aliev G, Nunomura A, Fujioka H, Russell RL, Atwood CS, Johnson AB, Kress $Y$, Vinters HV, Tabaton M, et al. Mitochondrial abnormalities in Alzheimer's disease. J Neurosci. 2001;21:3017-23.

77. Parker WD Jr, Filley CM, Parks JK. Cytochrome oxidase deficiency in Alzheimer's disease. Neurology. 1990;40:1302-3.

78. Perry EK, Perry RH, Tomlinson BE, Blessed G, Gibson PH. Coenzyme Aacetylating enzymes in Alzheimer's disease: possible cholinergic 'compartment' of pyruvate dehydrogenase. Neurosci Lett. 1980;18:105-10.

79. Gibson GE, Sheu KF, Blass JP, Baker A, Carlson KC, Harding B, Perrino P. Reduced activities of thiamine-dependent enzymes in the brains and peripheral tissues of patients with Alzheimer's disease. Arch Neurol. 1988;45: 836-40.

80. Sheehan JP, Swerdlow RH, Miller SW, Davis RE, Parks JK, Parker WD, Tuttle JB. Calcium homeostasis and reactive oxygen species production in cells transformed by mitochondria from individuals with sporadic Alzheimer's disease. J Neurosci. 1997;17:4612-22.

81. Schon EA, Shoubridge EA, Moraes CT. Cybrids in Alzheimer's disease: a cellular model of the disease? Neurology. 1998;51:326-7.

82. Swerdlow RH, Burns JM, Khan SM. The Alzheimer's disease mitochondrial cascade hypothesis. J Alzheimers Dis. 2010;20(Suppl 2):S265-79.

83. Swerdlow RH, Khan SM. A "mitochondrial cascade hypothesis" for sporadic Alzheimer's disease. Med Hypotheses. 2004;63:8-20.

84. Park JS, Davis RL, Sue CM. Mitochondrial dysfunction in Parkinson's disease: new mechanistic insights and therapeutic perspectives. Curr Neurol Neurosci Rep. 2018;18:21.

85. Jin H, Kanthasamy A, Ghosh A, Anantharam V, Kalyanaraman B, Kanthasamy AG. Mitochondria-targeted antioxidants for treatment of Parkinson's disease: preclinical and clinical outcomes. Biochim Biophys Acta. 1842;2014:1282-94.

86. Parkinson Study Group QEI, Beal MF, Oakes D, Shoulson I, Henchcliffe C, Galpern WR, Haas R, Juncos JL, Nutt JG, Voss TS, et al. A randomized clinical trial of high-dosage coenzyme Q10 in early Parkinson disease: no evidence of benefit. JAMA Neurol. 2014;71:543-52.

87. Writing Group for the NETiPDI, Kieburtz K, Tilley BC, Elm JJ, Babcock D, Hauser R, Ross GW, Augustine AH, Augustine EU, Aminoff MJ, et al. Effect of creatine monohydrate on clinical progression in patients with Parkinson disease: a randomized clinical trial. JAMA. 2015;313:584-93.

88. Chang JC, Wu SL, Liu KH, Chen YH, Chuang CS, Cheng FC, Su HL, Wei YH, Kuo SJ, Liu CS. Allogeneic/xenogeneic transplantation of peptide-labeled mitochondria in Parkinson's disease: restoration of mitochondria functions 
and attenuation of 6-hydroxydopamine-induced neurotoxicity. Transl Res. 2016;170:40-56 e3

89. Shi X, Zhao M, Fu C, Fu A. Intravenous administration of mitochondria for treating experimental Parkinson's disease. Mitochondrion. 2017;34:91-100.

90. Sims NR, Muyderman H. Mitochondria, oxidative metabolism and cell death in stroke. Biochim Biophys Acta. 1802;2010:80-91.

91. Huang PJ, Kuo CC, Lee HC, Shen Cl, Cheng FC, Wu SF, Chang JC, Pan HC, Lin SZ, Liu CS, Su HL. Transferring Xenogenic mitochondria provides neural protection against ischemic stress in ischemic rat brains. Cell Transplant. 2016;25:913-27.

92. Masuzawa A, Black KM, Pacak CA, Ericsson M, Barnett RJ, Drumm C, Seth P, Bloch DB, Levitsky S, Cowan DB, McCully JD. Transplantation of autologously derived mitochondria protects the heart from ischemiareperfusion injury. Am J Physiol Heart Circ Physiol. 2013;304:H966-82.

93. Kaza AK, Wamala I, Friehs I, Kuebler JD, Rathod RH, Berra I, Ericsson M, Yao $\mathrm{R}$, Thedsanamoorthy JK, Zurakowski D, et al. Myocardial rescue with autologous mitochondrial transplantation in a porcine model of ischemia/ reperfusion. J Thorac Cardiovasc Surg. 2017;153:934-43.

94. Gollihue JL, Patel SP, Eldahan KC, Cox DH, Donahue RR, Taylor BK, Sullivan PG, Rabchevsky AG. Effects of Mitochondrial Transplantation on Bioenergetics, Cellular Incorporation, and Functional Recovery after Spinal Cord Injury. J Neurotrauma. 2018:35(15):1800-18.

95. Taylor CA, Bell JM, Breiding MJ, Xu L. Traumatic brain injury-related emergency department visits, hospitalizations, and deaths - United States, 2007 and 2013. MMWR Surveill Summ. 2017;66:1-16.

96. Dewan MC, Rattani A, Gupta S, Baticulon RE, Hung YC, Punchak M, Agrawal A, Adeleye AO, Shrime MG, Rubiano AM, et al. Estimating the global incidence of traumatic brain injury. J Neurosurg. 2018:1-18.

97. Lifshitz J, Sullivan PG, Hovda DA, Wieloch T, McIntosh TK. Mitochondrial damage and dysfunction in traumatic brain injury. Mitochondrion. 2004;4: $705-13$

98. Singh IN, Sullivan PG, Deng Y, Mbye LH, Hall ED. Time course of posttraumatic mitochondrial oxidative damage and dysfunction in a mouse model of focal traumatic brain injury: implications for neuroprotective therapy. J Cereb Blood Flow Metab. 2006:26:1407-18.

99. Wu Q, Xia SX, Li QQ, Gao Y, Shen X, Ma L, Zhang MY, Wang T, Li YS, Wang ZF, et al. Mitochondrial division inhibitor 1 (Mdivi-1) offers neuroprotection through diminishing cell death and improving functional outcome in a mouse model of traumatic brain injury. Brain Res. 2016;1630:134-43.

100. Fu A, Shi X, Zhang H, Fu B. Mitotherapy for fatty liver by intravenous Administration of Exogenous Mitochondria in male mice. Front Pharmacol. 2017:8:241.

Ready to submit your research? Choose BMC and benefit from:

- fast, convenient online submission

- thorough peer review by experienced researchers in your field

- rapid publication on acceptance

- support for research data, including large and complex data types

- gold Open Access which fosters wider collaboration and increased citations

- maximum visibility for your research: over $100 \mathrm{M}$ website views per year

At $\mathrm{BMC}$, research is always in progress.

Learn more biomedcentral.com/submissions 